\title{
Cross-sectional Echocardiographic Observation of the Short Axis Views of the Left Ventricle in Hypertrophic Cardiomyopathy
}

\author{
Takaaki Isshiki, M.D., Toru UmEdA, M.D., \\ and Kiyoshi MACHII, M.D.
}

SUMmary

Short axis vicws of the left ventricle were studied in 68 paticnts with hypertrophic cardiomyopathy (HCM) and 20 healthy persons using crosssectional echocardiography.

Configurations of the short axis view of the left ventricular cavity at the level of the papillary muscles in HCM were classified into 2 types, dumbbell and mushroom, which were formed by the position of papillary muscles. Anterior dislocation of the papillary muscles was seen in the dumbbell type, and marked hypertrophy of the papillary muscles in the proper position was seen in the mushroom type. Dumbbell shape (50 patients) was observed in the patients with marked hypertrophy in the basal portion of interventricular septum. Mushroom shape (18 patients) was seen in the patients with hypertrophy around the apex of the heart except for in a few patients.

Areas of the papillary muscles (AP) and the left ventricular cavity (ALV) were measured and AP/ALV + AP ratio was calculated. The $\mathrm{AP} / \mathrm{ALV}+\mathrm{AP}$ ratio in $\mathrm{HCM}$ was significantly larger than that in the healthy persons, but no difference was seen between these 2 groups of HCM.

It should be noted that the position of the hypertrophied papillary muscles is closely related to the distribution of hypertrophy in HCM.

\section{Additional Indexing Words :}

Papillary muscles Asymmetric septal hypertrophy Apical hypertrophy Distribution of hypertrophy

THE word " asymmetric septal hypertrophy (ASH)" has been used as a 1 synonym of hypcrtrophic cardiomyopathy (HCM) for several years, ${ }^{1)-3}$ ) but recently, some ideas opposite to the concept of ASH were brought out, ${ }^{4), 5}$, showing that hypertrophy in HCM could be seen in every portion of the heart. As far as the short axis views of the left ventricle in HCM are concerned, minute observation has not yet been achieved.

From the Center for Cardiovascular Disease, Mitsui Memorial Hospital, 1 Kanda-Izumi-cho, Chiyoda-ku, Tokyo 101, Japan.

Received for publication October 12, 1979. 
Our study was designed to make minute observation of the left ventricular cavity and the papillary muscles by the short axis view of cross-sectional echocardiogram, and to clarify the particular relationship between the position of papillary muscles and the distribution of hypertrophy in HCM.

\section{Materials and Methods}

Sixty-eight patients with HCM aged 20 to 79 ( 56 males, 12 females), and 20 healthy persons aged 23 to 35 , including 1 female, with no symptoms or signs referable to the cardiovascular system were observed (Table I).

HCM was defined as the left ventricular hypertrophy of unknown origin. The patients were separated into 2 groups by the distribution of hypertrophy. Interventricular septums with the basal portion thickness of more than $15 \mathrm{~mm}$ (including $15 \mathrm{~mm}$ ) were defined as Group $\mathrm{A}$, and those with less than $15 \mathrm{~mm}$ were defined as Group B. Basal portion thickness of the interventricular septum was measured at $10 \mathrm{~mm}$ from the aorto-septal junction in end-diastole coinciding with the peak of $\mathrm{R}$ wave in electrocardiogram (Fig. 1). In 68 patients, 52 were put in

Table I. Materials

\begin{tabular}{c|c|c|c}
\hline Condition & No. of patients & Age & Sex \\
\hline $\begin{array}{c}\text { Hypertrophic } \\
\text { cardionyopathy }\end{array}$ & 68 & & \\
Group A & 52 & $20-79$ & M: 42, F: 10 \\
Group B & 16 & $31-72$ & M: 14, F: 2 \\
Normal & 20 & $23-35$ & M: 19, F: 1
\end{tabular}

$\mathrm{M}=$ male $; \mathrm{F}=$ female.

Group A: Patients with hypertrophic cardiomyopathy whose basal portion thickness of the interventricular septum is more than $15 \mathrm{~mm}$ (including $15 \mathrm{~mm}$ ). Group B: Patients with hypertrophic cardiomyopathy whose basal portion thickness of the interventricular septem is less than $15 \mathrm{~mm}$.

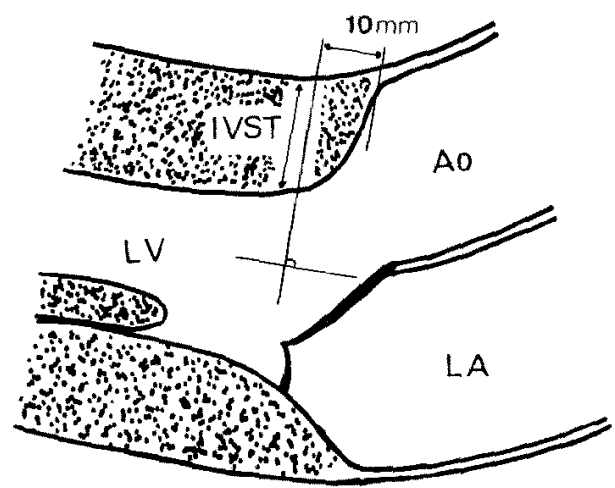

Fig. 1. Schematic illustration of a long axis view of the left ventricle. Basal portion thickness of the interventricular septum is measured at $10 \mathrm{~mm}$ from the aorto-septal junction in end-diastole. IVST =interventricular septal thickness; $L V=$ lefi ventricle; $L A=$ left atrium; $A O=$ aorta. 
Group A, and the other 16 Group B. The ASH patients were included in Group $A$, and the patients with hypertrophy of both interventricular septum and posterior wall, which was not thought to be ASH, for example, were also included in Group A.

The commercially available Toshiba SSH-11A, real-time phased-array sector scanner was used and cross-sectional images were recorded on video tape for minute observation. The stopped images by Honeywell hard copy system were recorded for measuring.

Three different short axis views of the left ventricle were obtained from the third or the fourth intercostal space along the left sternal border: 1) The papillary

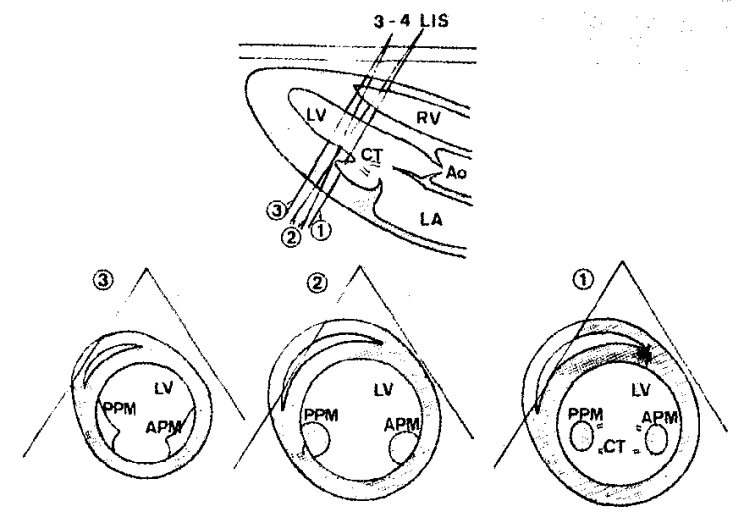

Fig. 2. Short axis views of the left ventricle at the level of the anterior and posterior papillary muscles. 1) The papillary muscles are apart from the left ventricular wall. 2) The papillary muscles are in contact with the left ventricular wall. 3) Roots of the papillary muscles are seen in this short axis view. $A P M=$ anterior papillary muscle; $P P M=$ posterior papillary muscle; $\mathrm{CT}=$ chordae tendineae; $\mathrm{LV}=$ left ventricle; $\mathrm{RV}=$ right ventricle; $\mathrm{LA}=$ left atrium; $A o=$ aorta.

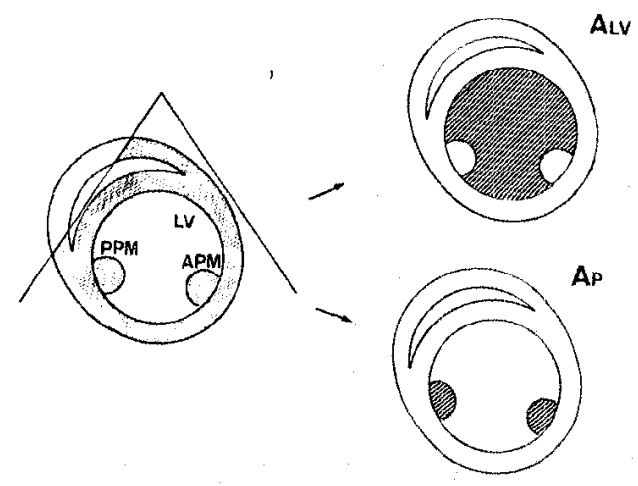

Fig. 3. Areas of the papillary muscles (AP) and left ventricular cavity (ALV) in the short axis view at the level of the papillary muscles. The papillary muscles were separated from the left ventricular wall by imaginary lines. 
muscles are free from the left ventricular walls, and the chordae tendineae can be seen at the same time. 2) The chordae tendineae cannot be seen and the papillary muscles are in contact with the left ventricular wall. 3) An image is slightly nearer to the apex of the heart and roots of the papillary muscles are seen in this short axis view (Fig. 2). In this entire study, the second image was used because this image is easier to obscrve, record and measure than the other 2.

In order to estimate the degree of hypertrophy of the papillary muscles, the areas of the papillary muscles (AP) and the left ventricular cavity (ALV) were measured at end-systole and end-diastole, and the AP/ALV + AP ratio was calculated (Fig. 3). The papillary muscles were separated from the left ventricular wall by imaginary lines that were extended from the endocardium. Twenty-seven patients and 17 healthy persons were measured and calculated. In this procedure, the patients with marked hypertrophy whose left ventricular cavities were almost completely obliterated in end-systole were picked out because it was hard to measure the areas of any apparatus in end-systole.

\section{ResUlts}

In patients with HCM, 2 particular shapes of the left ventricular cavity were observed, dumbbell and mushroom. Dumbbell shape was formed by
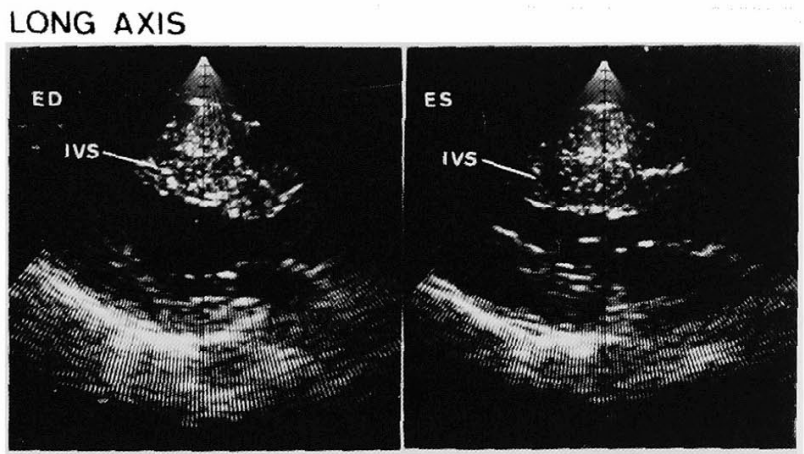

\section{SHORT AXIS}
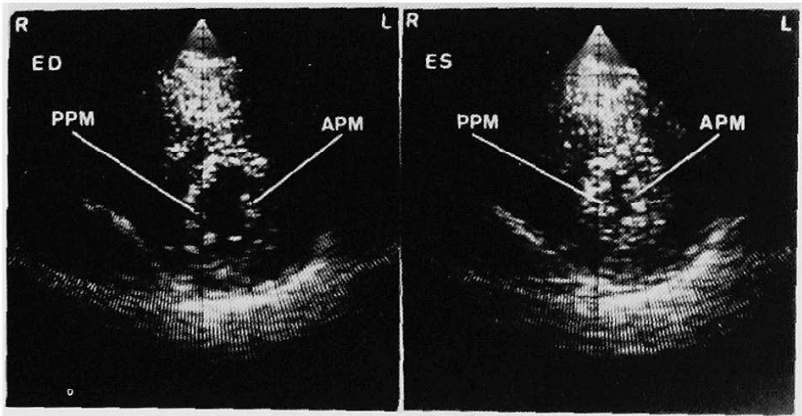

Fig. 4. Cross-sectional echocardiograms of a patient in Group A HCM. Hypertrophy is confined to the interventricular septum and the papillary muscles, which obliterate the left ventricular cavity during systole. The left ventricular cavity shows a dumbbell shape in the short axis view. 

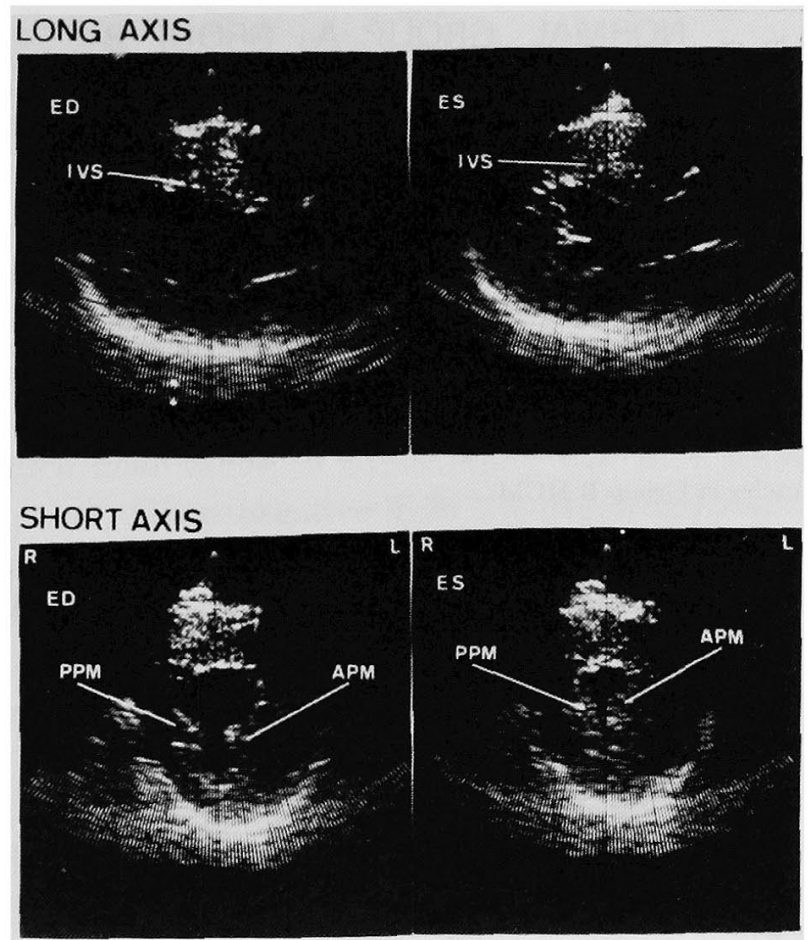

Fig. 5. Gross-sectional echocardiograms of a patient in Group B HCM. Hypertrophy is confined to the apical portion of the left ventricle and the papillary muscles, which obliterate the posterior half of the left ventricular cavity during systole. The left ventricular cavity shows a mushroom shape in the short axis view.

the anterior dislocation of the hypertrophied anterior and posterior papillary muscles in the oval shaped left ventricular cavity. The difference of the degree of hypertrophy between the anterior and the posterior papillary muscles was observed in several patients. Mushroom shape was formed by markedly hypertrophied left ventricular wall and the papillary muscles. The papillary muscles were not dislocated, but almost the entire posterior half of the left ventricular cavity was occupied by the hypertrophied papillary muscles (Figs. 4, 5).

Dumbbell shape was seen in all Group A patients except for in 2. Both of these 2 patients showed hypertrophy of the interventricular septum from the basal to the apical portion of the heart. Mushroom shape was seen in all Group B patients without exception (Fig. 6).

The results of measurements are as follows (Fig. 7). AP in Group B patients was significantly larger than that in the healthy persons $(p<0.001)$, but those in Group A patients showed no significant difference. ALVs in both groups were significantly smaller than that in the healthy persons $(p<$ 
NORMAL GROUP A GROUP B
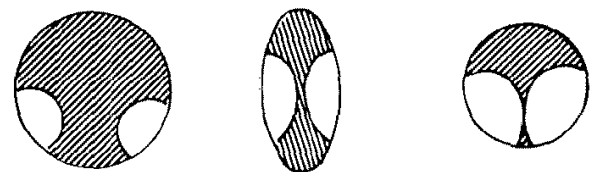

DUMBBELL MUSHROOM

Fig. 6. Configurations of the left ventricular cavity. The papillary muscles are dislocated anteriorly in the oval shaped left ventricular cavity in Group A HCM. No dislocation of the papillary muscles is seen, but the posterior half of the left ventricular cavity is occupied by hypertrophied papillary muscles in Group B HCM.
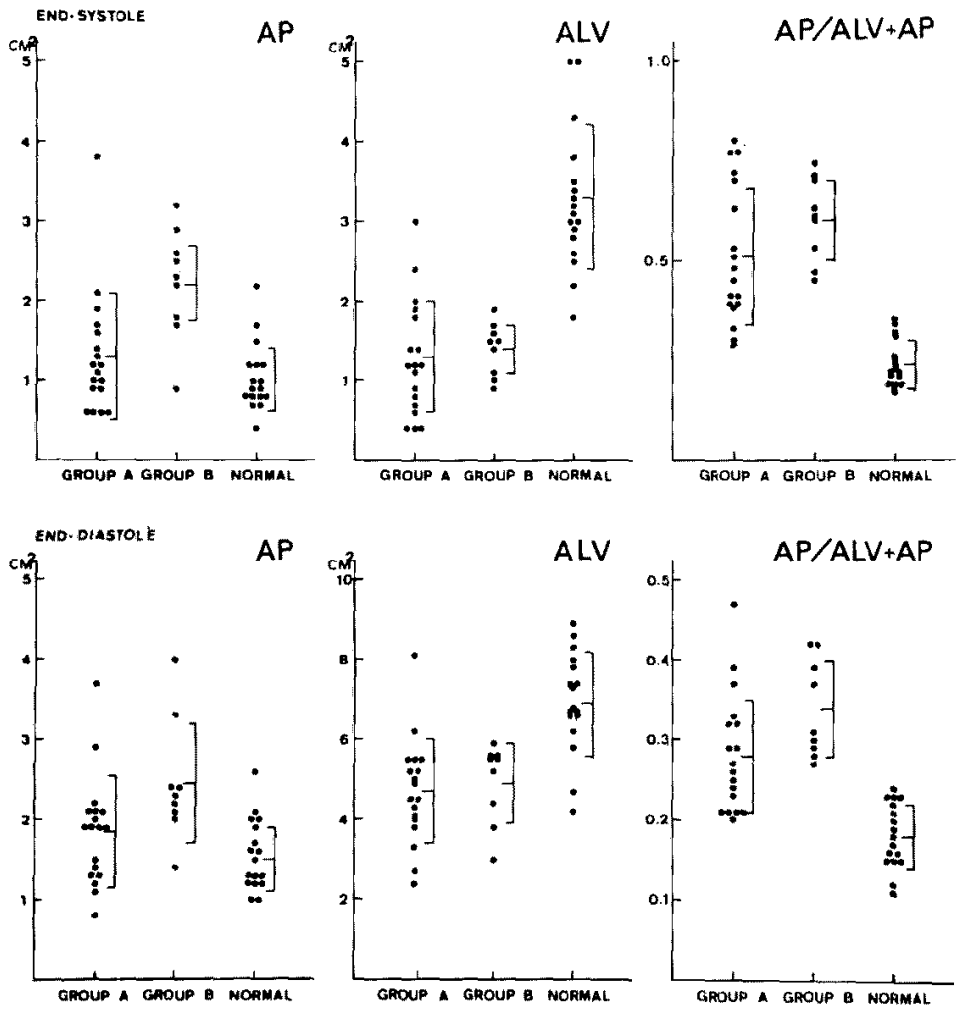

Fig. 7. Areas of the papillary muscles (AP), left ventricular cavity (ALV), and calculated AP/ALV + AP ratios are compared. AP/ALV + AP ratios of HCM are significantly larger than controls, but no difference is seen between 2 groups of HCM.

0.001). Calculated AP/ALV + AP ratios of HCM were significantly larger $(p<0.001)$, but no difference could be obtained between these 2 types of HCM. These results were more significant in end-systole. 


\section{Discussion}

HCM has been clinically noted since 1960 , when Goodwin et a ${ }^{6)}$ named hypertrophic obstructive cardiomyopathy (HOCM) and Braunwald et al ${ }^{7}$ named idiopathic hypertrophic subaortic stenosis (IHSS). Henry et al ${ }^{11}$ insisted on calling this disease ASH, in 1973, studied by M-mode echocardiography and pathological observations. Recently, many patients with HCM which was not thought to be ASH have been observed by cross-sectional echocardiography. ${ }^{4,8}, \quad$ Apical hypertrophy was reported as one of the other types of HCM, in which distribution of hypertrophy was seen around the apical portion of the heart. Giant negative $\mathrm{T}$ in left precordial leads with normal electrical axis in electrocardiogram was reported as another characteristic feature of the disease. ${ }^{9,10)}$ Hypertrophy should occur in every portion of the heart, ${ }^{4}$ ' and we should avoid using the word "ASH " as a synonym of HCM. ${ }^{5 \text { " }}$

In this study, 39 patients were thought to have ASH, 2 patients showed hypertrophy of the posterior wall as well as of the interventricular sptum, 16 patients showed hypertrophy around the apex of the heart (apical hypertrophy), and 11 patients showed hypertrophy around the apex as well as of the interventricular septum. No patient was seen who showed hypertrophy only in the posterior wall or the free wall. To simplify this complicated situation, the classification by the basal portion thickness of $15 \mathrm{~mm}$ was made in this study. In 52 patients of Group A HCM, 2 without ASH were included, and all of Group B HCM were thought to have apical hypertrophy in this study,

The most remarkable finding of the short axis views of the left ventricle was the difference in the position of the hypertrophied papillary muscles. The papillary muscles were dislocated anteriorly to the middle of the left ventricular cavity making dumbbell shape in Group A HCM. The left ventricular cavity was compressed by both the interventricular septum and the free wall. On the contrary, the dislocation of the papillary muscles was not seen but marked hypertrophy of the papillary muscles, making mushroom shape, was seen in Group B HCM. Several patients showed intermediate shapes. For example, slight anterior dislocation of the papillary muscles was observed in a few patients of Group B HCM. Some patients showed a difference in the degree of hypertrophy between the anterior and posterior papillary muscles. The left ventriculograms of the 2 particular types of HCM also showed significant difference. RAO views of the left ventriculogram reveal a banana-like shape in end-systole in Group $\mathrm{A} \mathrm{HCM}^{2}$ and a spadelike shape in end-diastole in Group $\mathrm{B} \mathrm{HCM}^{\mathrm{v}}$ (Fig. 8). This difference is mainly due to the distribution of hypertrophy. 

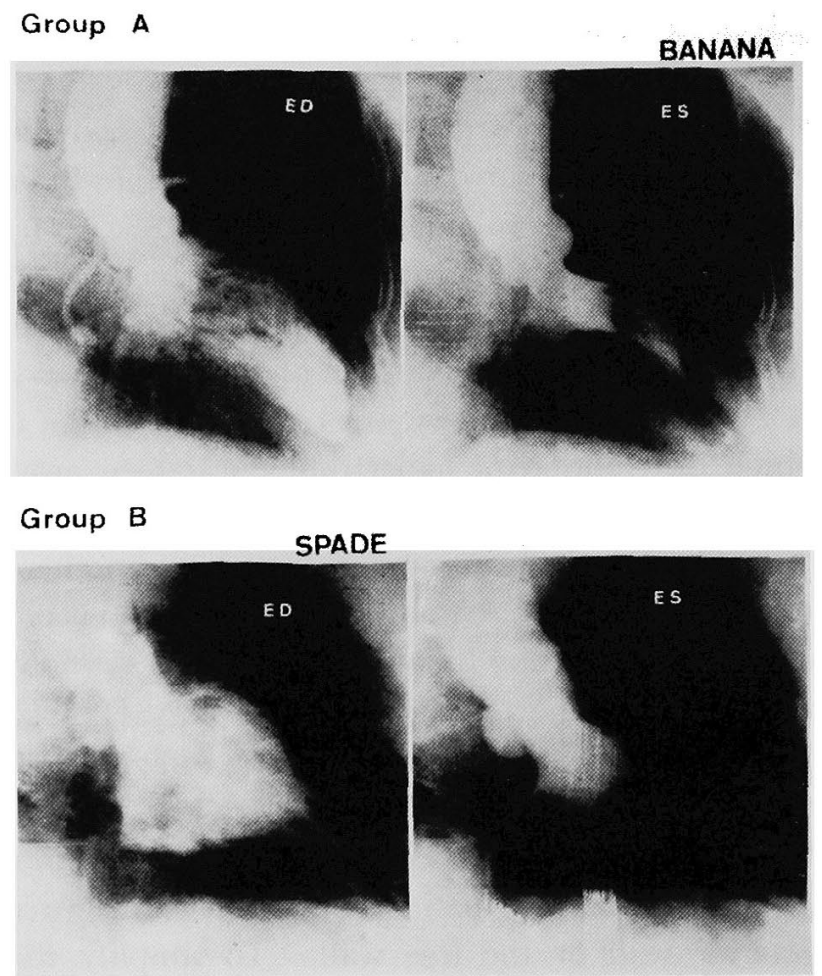

Fig. 8. Left ventriculograms at end-systole and end-diastole in the RAO projection of patients in Group A and Group B HCM. Banana-like shape is seen in end-systole in Group A patient. Spade-like shape is seen in enddiastole in Group B patient.

The cause of this particular relation between the short axis view and the distribution of hypertrophy is not known exactly. It is speculated that, in Group A HCM, the anterior part of the left ventricular cavity is occupied by the hypertrophied interventricular septum, and the remaining part of the left ventricular cavity is compressed by the interventricular septum and the free wall resulting in the relatively anterior position of the papillary muscles. In Group B HCM, no deformity of the left ventricular cavity is seen, and the papillary muscles are seen in the proper position, but extreme hypertrophy accompanied by abnormal thickening around the apex of the heart. It would be very important to note that this simple and easy classification by the basal portion thickness of interventricular septum resulted in showing the difference in the position of the papillary muscles. Whether or not this particular classification is related to some clinical findings, the cause of the systolic anterior motion in echocardiogram, for example, would be a very interesting problem to be studied.

As far as the AP and ALV are concerned, the calculated AP/ALV + AP 
ratio should be useful for detecting and estimating HCM, because that in both groups of HCM was significantly larger than that in the healthy persons. However, these data have to be used for reference, because some inevitable errors of this method in measuring cannot be ignored. It will be sometimes difficult to measure on the stopped images by the hard copy system. Imaginary lines between the papillary muscles and the left ventricular wall will also be the cause for misconception of the areas.

\section{REFERENCES}

1. Henry WL, Clark CE, Epstein SE: Asymmetric septal hypertrophy. Echocardiographic identification of the pathognomonic anatomic abnormality of IHSS. Circulation 47: 225, 1973

2. Henry WL, Clark CE, Epstein SE: Asymmetric septal hypertrophy (ASH). The unifying link in the IHSS disease spectrum observations regarding its pathogenesis, pathophysiology and course. Circulation 47: 827, 1973

3. Henry WL, Clark CE, Roberts WC, Morrow AG, Epstein SE: Differences in distribution of myocardial abnormalities in patients with obstructive and nonobstructive asymmetric septal hypertrophy (ASH). Echocardiographic and gross anatomic findings. Circulation 50: 447 , 1974

4. Nimura Y, Sakakibara H, Matsuo H, Matsumoto M, Miyatake K, Nagata S, Beppu S, Tamai $M$, Senda $S$, Sato $K$, Abe H: Studies on left ventricular anatomy and events in hypertrophic cardiomyopathy with ultrasono-cardiotomography in special reference to obstructive signs. Heart 7: 1461, 1975 (in Japanese)

5. Maron BJ, Epstein SE: Hypertrophic cardiomyopathy. A discussion of nomenclature. Am J Cardiol 43: 1242, 1979

6. Goodwin JF, Hollman A, Cleland WP, Teare D: Obstructive cardiomyopathy simulting aortic stenosis. Brit Heart J 22: 403, 1960

7. Braunwald E, Morrow AG, Cornell WP, Aygen MM, Hilbish EF: Idiopathic hypertrophic subaortic stenosis. Clinical, hemodynamic and angiographic manifestations. Am J Med 29: 924,1960

8. Falicov RE, Resnecov L, Bharati S, Lev M: Mid-ventricular obstruction. A valiant of obstructive cardiomyopathy. Am J Cardiol 37: 432, 1976

9. Yamaguchi $H$, Ishimura $T$, Nishiyama $S$, Nagasaki $F$, Nakanishi $S$, Nishijo $T$, Umeda $T$, Machii K: Hypertrophic nonobstructive cardiomyopathy with giant negative $T$ waves (Apical hypertrophy). Ventriculographic and echocardiographic features in 30 patients. Am J Cardiol 44: 401, 1979

10. Sakamoto T, Tei G, Murayama M, Ichiyasu H, Hada Y, Hayashi T, Amano K: Giant $T$ inversion as a manifestation of asymmetric apical hypertrophy (AAH) of the left ventricle. Echocardiographic and ultrasono-cardiotomographic study. Jap Heart J 17: 611, 1976 